\title{
Vibrations of a Horizontal Elastic Band Plate Submerged in Fluid of Constant Depth
}

\author{
Kazimierz Szmidt, Benedykt Hedzielski \\ Institute of Hydro-Engineering, Polish Academy of Sciences, ul. Kościerska 7, 80-328 Gdańsk, Poland, \\ e-mail: jks@ibwpan.gda.pl
}

(Received October 26, 2016; revised January 10, 2017)

\begin{abstract}
The paper deals with free and forced vibrations of a horizontal thin elastic plate submerged in an infinite layer of fluid of constant depth. In free vibrations, the pressure load on the plate results from assumed displacements of the plate. In forced vibrations, the fluid pressure is mainly induced by water waves arriving at the plate. In both cases, we have a coupled problem of hydrodynamics in which the plate and fluid motions are coupled through boundary conditions at the plate surface. At the same time, the pressure load on the plate depends on the gap between the plate and the fluid bottom. The motion of the plate is accompanied by the fluid motion. This leads to the so-called co-vibrating mass of fluid, which strongly changes the eigenfrequencies of the plate. In formulation of this problem, a linear theory of small deflections of the plate is employed. In order to calculate the fluid pressure, a solution of Laplace's equation is constructed in the doubly connected infinite fluid domain. To this end, this infinite domain is divided into sub-domains of simple geometry, and the solution of the problem equation is constructed separately for each of these domains. Numerical experiments are conducted to illustrate the formulation developed in this paper.
\end{abstract}

Key words: elastic plate, free vibrations, forced vibrations, eigenfrequencies, co-vibrating mass of fluid

\section{Introduction}

In offshore engineering, we frequently deal with the problem of water flow-induced loads on structures. Hydrodynamic forces depend on fluid flows in the vicinity of the structure as well as on the structure size, shape, rigidity and foundation. Usually, such a structure consists of parts of simple geometry such as bars, pipes and plates, and therefore, in a theoretical description of the structure dynamics, it is reasonable to investigate a dynamic behaviour of individual elements. Among them, of primary importance are elastic plates submerged in fluid and loaded with forces induced by gravitational waves. An example is a horizontal plate foundation of a windmill installed in a sea coastal zone. Usually, hydrodynamic forces depend not only on the 
waves themselves, but also on the foundation of the plate and its orientation to the direction of wave propagation. For instance, wave forces on a plane plate perpendicular to the wave propagation direction are different from those for a plate whose surface is parallel to the wave direction. In general, these forces may also depend on the distance between the plate and the boundaries of the fluid domain. In cases of horizontal plates placed at a small distance from the sea bottom, one may expect a certain amplification of hydrodynamic forces loading these plates. This phenomenon is associated with changes in the velocities in flows on the upper and bottom surfaces of the plates. At the same time, a vibration of the plate submerged in fluid leads to the so-called co-vibrating mass of fluid, which strongly changes the eigenfrequencies of this plate.

With respect to the above, we focus our investigations on the coupled hydrodynamic problem of a horizontal plate vibrating in a layer of fluid of constant depth. In order to simplify our discussion, we confine our attention to a simply supported elastic band plate, which makes it possible to reduce the description of a physical three-dimensional problem to a two-dimensional one. In a formal way, the two-dimensional description model corresponds directly to a simply supported horizontal beam, submerged in fluid of constant depth. An additional simplification introduced into the description is that plate deflections are assumed infinitesimally small. In theoretical investigations, we resort to approximate modeling that can describe the main features of this phenomenon.

As regards vibrations of plates in contact with fluid, Solecki (1966) discussed the problem of an infinite plate floating on a water half-space. A similar problem of the deformation of floating ice plates was investigated by Kerr and Palmer (1972). As far as a finite fluid body is concerned, Sawicki (1975) discussed the problem of the dynamics of floating roofs of cylindrical tanks. A detailed discussion on the dynamics of an elastic band plate floating on a tank with a rectangular cross section is given in Sawicki (1976). In particular, general solutions for the problem of free and forced vibrations of the plate may be found in that paper. The problem discussed in the present paper corresponds in a sense to that of Sawicki, but it deals with an infinite fluid domain and a fully submerged plate. Our main goal is to calculate a set of the lowest eigenfrequencies of the plate, dependent on the width of the gap between the plate and the bottom, as well as to evaluate deflections of the plate loaded with surface gravitational waves.

\section{Problem Formulation}

Let us consider the two-dimensional problem of a thin elastic band plate submerged in fluid, as shown schematically in Fig. 1.

The motion of the plate is accompanied by the fluid motion, and thus we have the so-called coupled problem of hydrodynamics. This coupling takes place through the boundary conditions at the upper and bottom surfaces of the plate. The normal components of the fluid and plate velocities should be equal to each other. With respect 


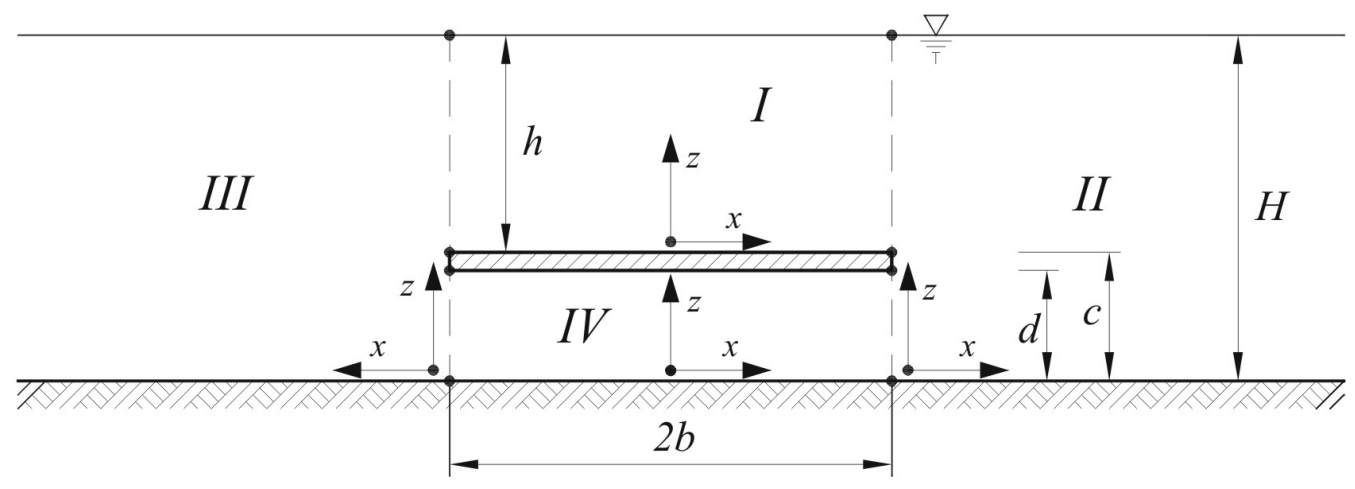

Fig. 1. Elastic plate submerged in a layer of fluid

to small deflections of the plate, its motion is governed by the following equation (Nowacki 1972):

$$
m_{p l .} \frac{\partial^{2} w}{\partial t^{2}}+D^{*} \frac{\partial^{4} w}{\partial x^{4}}=p_{\text {low. }}-p_{\text {upp. }},
$$

where $m_{p l}$. is the mass per unit width and length of the plate, $D^{*}=E \delta^{3} / 12\left(1-v^{2}\right)$ is the flexural rigidity of the plate ( $\delta$ is the plate thickness, and $v$ is Poisson's ratio), $p_{\text {low. }}$. and $p_{\text {upp. }}$. denote fluid pressure at the lower and upper surfaces of the plate. It should be stressed that the 'density' of the plate $m_{p l}=\left(\rho_{p l}-\rho\right) \delta$, where $\rho_{p l}$. is the density of the plate material, and $\rho$ is the fluid density. Assuming a potential velocity of the fluid motion, the associated fluid pressure is described by the formula

$$
p=-\rho \frac{\partial \Phi}{\partial t}
$$

where $\Phi(x, z, t)$ is the velocity potential satisfying Laplace's equation

$$
\frac{\partial^{2} \Phi}{\partial x^{2}}+\frac{\partial^{2} \Phi}{\partial z^{2}}=\nabla^{2} \Phi=0
$$

within the fluid domain and appropriate boundary conditions at the fluid boundaries.

In discussing the problem of free vibrations of the plate and, in particular, the problem of the plate eigenfrequencies when the fluid flow is induced solely by the motion of the plate, it is justified to assume that the free surface of the fluid is flat over the entire range of time considered (fluid pressure is constant at $z=H$ ). With this assumption, the plate-fluid system is conservative, i.e. there is no damping of the plate motion. When vibrations of the plate are forced, for instance by gravitational waves arriving from infinity, the potential function within the fluid domain is affected by vibrations of the plate. In the cases of both free and forced vibrations of the plate, it is necessary to solve the coupled problem of the plate and fluid motion. Before doing that, however, it is convenient to consider, in the first step, the simplest case of free 
vibrations of the plate in air. For such a case, equation (1) reduces to the following one:

$$
m_{p l} \frac{\partial^{2} w}{\partial t^{2}}+D^{*} \frac{\partial^{4} w}{\partial x^{4}}=0
$$

where $m_{p l .}=\rho_{p l} \delta$.

For harmonic vibrations, the following relation holds:

$$
w(x, t)=W(x) \exp (i \omega t) .
$$

Substitution of this relation into equation (4) gives

$$
-\omega^{2} m_{p l .} W+D^{*} \frac{\partial^{4} W}{\partial x^{4}}=0 .
$$

The deflection amplitude $W(x)$ is expressed in the form

$$
W(x)=\sum_{n} A_{n} \sin r_{n}(x+b), \quad r_{n}=\frac{n \pi}{2 b}, n=1,2, \cdots,
$$

which satisfies boundary conditions at $x= \pm b$. From substitution of this solution into (6), the following is obtained:

$$
\sum_{n} A_{n}\left[-m_{p l .} \omega^{2}+D^{*}\left(r_{n}\right)^{4}\right] \sin r_{n}(x+b)=0 .
$$

It may be seen that the functions $\sin r_{n}(x+b)(n=1,2, \cdots)$ form the eigenfunction set of the problem. At the same time, equation (8) leads to the set of eigenfrequencies of the plate

$$
\omega_{n}=\left(\frac{n \pi}{2 b}\right)^{2} \sqrt{\frac{D^{*}}{m_{p l} .}}, \quad n=1,2, \cdots .
$$

In order to find an associated set of frequencies for the case of free vibrations of the plate in fluid, it is necessary to calculate the fluid pressure and to solve equation (1). The plate deflection for this case is expressed in the form of a series with respect to eigenfunctions $\sin r_{n}(x+b)$, inherent for vibrations in air. To calculate the fluid pressure, we have to find a solution of Laplace's equation in the doubly connected fluid domain, satisfying the following system of boundary conditions:

$$
\begin{aligned}
& \left.\Phi\right|_{x \rightarrow \pm \infty}=0,\left.\quad \frac{\partial \Phi}{\partial x}\right|_{x \rightarrow \pm \infty}=0,\left.\quad \frac{\partial \Phi}{\partial z}\right|_{z=0}=0, \\
& \left.\frac{\partial \Phi}{\partial n} \simeq \frac{\partial \Phi}{\partial z}\right|_{\text {upp.bot. }}=\frac{\partial w(x, t)}{\partial t}=\dot{w}(x, t),
\end{aligned}
$$

where 'upp.bot.' means the upper and bottom surfaces of the plate.

For the thin plate considered, the normal velocity components of the fluid at two sides of the plate (upper and bottom surfaces) are assumed to be equal to the transverse 
velocity of the plate centre. A remark is needed. In addition to the boundary conditions given above, it is necessary to investigate the potential behaviour at the plate end points $(x= \pm b)$. If the cross section of the plate is rectangular with two right angles at these ends, the fluid velocity field is singular at these corner points. It may be shown, however, that this fluid velocity field is an integrable function along an arbitrary path in the vicinity of the end points.

In order to find a solution of Laplace's equation in the doubly connected fluid domain, we divide this domain into four parts: $I, I I, I I I$ and $I V$ (see Fig. 1). In descriptions of the potential functions within these domains, it is convenient to introduce local Cartesian coordinate systems. Thus, with respect to these coordinate systems, the general solution of Laplace's equation read:

Subdomain III $(0 \leq x<\infty, \quad 0 \leq z \leq H)$

$$
\phi(x, z, t)=-\sum_{j=1} C_{j}(t) \frac{1}{k_{j}} \exp \left(-k_{j} x\right) \cos k_{j} z, \quad k_{j}=\frac{2 j-1}{2 H} \pi, \quad j=1,2, \cdots .
$$

Subdomain $I I(0 \leq x<\infty, \quad 0 \leq z \leq H)$

$$
\phi(x, z, t)=-\sum_{j=1} B_{j}(t) \frac{1}{k_{j}} \exp \left(-k_{j} x\right) \cos k_{j} z, \quad k_{j}=\frac{2 j-1}{2 H} \pi, \quad j=1,2, \cdots .
$$

Subdomain $I(-b \leq x<+b, \quad 0 \leq z \leq h)$

$$
\begin{aligned}
& \phi(x, z, t)=-\sum_{n=1} \dot{A}_{n}(t) \frac{1}{r_{n}} \frac{1}{v_{n}}\left[\exp \left(-r_{n} z\right)-\exp r_{n}(z-2 h)\right] \sin r_{n}(x+b)+ \\
& +\sum_{m=1}\left[D_{m}^{1}(t) \frac{\cosh \left(k_{m}^{*} x\right)}{\cosh \left(k_{m}^{*} b\right)}+D_{m}^{2}(t) \frac{\sinh \left(k_{m}^{*} x\right)}{\sinh \left(k_{m}^{*} b\right)}\right] \cos k_{m}^{*} z, \quad r_{n}=\frac{n \pi}{2 b}, \\
& v_{n}=1+\exp \left(-2 r_{n} h\right), \quad n=1,2, \cdots, \quad k_{m}^{*}=\frac{2 m-1}{2 h} \pi, \quad m=1,2, \cdots,
\end{aligned}
$$

where $\dot{A}_{n}(t)=d A_{n} / d t$.

Subdomain $I V(-b \leq x \leq+b, \quad 0 \leq z \leq d)$

$$
\begin{aligned}
\varphi(x, z, t) & =\sum_{n=1} \dot{A}_{n}(t) \frac{1}{r_{n}} \frac{\cosh r_{n} z}{\sinh r_{n} d} \sin r_{n}(x+b)+ \\
& +E_{0}(t)+\sum_{m=1}\left[E_{m}^{1}(t) \frac{\cosh k_{m} x}{\cosh k_{m} b}+E_{m}^{2}(t) \frac{\sinh k_{m} x}{\sinh k_{m} b}\right] \cos k_{m} z \\
& r_{n}=\frac{n \pi}{2 b}, \quad n=1,2, \cdots, \quad k_{m}=\frac{m \pi}{d}, \quad m=1,2, \cdots
\end{aligned}
$$

One can see that the series in the infinite domains (equations 11 and 12) quickly decay as $x \rightarrow \infty$. In practical calculations, it is justified to neglect the series for $x \geq L$, where $L$ may be specified for a particular fluid motion considered. In this way, the 
solution in the finite fluid domain $(0 \leq x \leq L)$ will be practically equal to that valid in the infinite domain $(0 \leq x \leq \infty)$. To save space, hereinafter we omit the time character $t$ in description of the functions $\dot{A}(t), \cdots, E(t)$, i.e. all functions are named constants. In accordance with the linear problem considered, all constants, $B_{j}, C_{j}, j=1,2, \cdots$, $D_{m}^{1}, D_{m}^{2}, m=1,2, \cdots$ and $E^{0}$, and $E_{m}^{1}, E_{m}^{2}, m=1,2, \cdots$, may be expressed in terms of the constants $\dot{A}_{n}, \quad n=1,2, \cdots$. It means that, for an arbitrary deflection of the plate, it is possible to find appropriate solutions within the corresponding fluid domains. To this end, we match the solutions at common boundaries of the subdomains. Thus, let us assume that, in advance, the solutions corresponding to $B_{j}$ and $C_{j}(j=1,2, \cdots)$ are known. The potential $\varphi(x=b, z)$ below the plate should be equal to that of the right-hand side domain, i.e. to $\phi(x=0, z)$ at the common boundary. This condition gives

$$
E_{0}+\sum_{m=1}\left(E_{m}^{1}+E_{m}^{2}\right) \cos k_{m} z=-\sum_{j=1} B_{j} \frac{1}{k_{j}} \cos k_{j} z
$$

In a similar way, at the boundary $(x=-b, z)$ we have

$$
E_{0}+\sum_{m=1}\left(E_{m}^{1}-E_{m}^{2}\right) \cos k_{m} z=-\sum_{j=1} C_{j} \frac{1}{k_{j}} \cos k_{j} z .
$$

Multiplication of equation (17) in succession by $\cos k_{m} z \quad(m=1,2, \cdots)$ and then integration in the range $(0 \leq z \leq d)$ leads to the following formulae:

$$
\begin{aligned}
& E_{0}=-\frac{1}{d} \sum_{j=1} B_{j} \frac{1}{\left(k_{j}\right)^{2}} \sin k_{j} d, \quad k_{j}=\frac{2 j-1}{2 H} \pi, k_{m}=\frac{m \pi}{d}, \\
& E_{m}^{1}+E_{m}^{2}=-\frac{2}{d} \sum_{j=1} B_{j}\left[\begin{array}{l}
\frac{(-1)^{m} \sin k_{j} d}{\left(k_{j}\right)^{2}-\left(k_{m}\right)^{2}} \text { for } k_{j} \neq k_{m} \\
\frac{d}{2 k_{j}} \quad \text { for } k_{j}=k_{m}
\end{array} \quad, j, m=1,2, \cdots\right.
\end{aligned}
$$

Similar results hold for the left boundary:

$$
\begin{aligned}
& E_{0}=-\frac{1}{d} \sum_{j=1} C_{j} \frac{1}{\left(k_{j}\right)^{2}} \sin k_{j} d, \\
& E_{m}^{1}-E_{m}^{2}=-\frac{2}{d} \sum_{j=1} C_{j}\left[\begin{array}{l}
\frac{(-1)^{m} \sin k_{j} d}{\left(k_{j}\right)^{2}-\left(k_{m}\right)^{2}} \text { for } k_{j} \neq k_{m} \\
\frac{d}{2 k_{j}} \text { for } k_{j}=k_{m}, \quad j, m=1,2, \cdots
\end{array}\right.
\end{aligned}
$$

The same procedure is employed for the upper fluid. Simple manipulations give

$$
\begin{aligned}
& \sum_{m=1}\left(D_{m}^{1}+D_{m}^{2}\right) \cos k_{m}^{*} z=-\sum_{j} B_{j} \frac{1}{k_{j}} \cos k_{j}(z+c), \\
& \sum_{m=1}\left(D_{m}^{1}-D_{m}^{2}\right) \cos k_{m}^{*} z=-\sum_{j} C_{j} \frac{1}{k_{j}} \cos k_{j}(z+c),
\end{aligned}
$$


and, finally

$$
\begin{aligned}
& D_{m}^{1}+D_{m}^{2}=-\frac{2}{h} \sum_{j=1} B_{j}\left[\begin{array}{l}
\frac{\sin k_{j} c}{\left(k_{m}^{*}\right)^{2}-\left(k_{j}\right)^{2}} \text { for } k_{j} \neq k_{m}^{*}, \\
\frac{1}{2\left(k_{j}\right)^{2}}\left(k_{j} h \cdot \cos k_{j} c-\sin k_{j} c\right) \text { for } k_{j}=k_{m}^{*},
\end{array}\right. \\
& k_{m}^{*}=\frac{(2 m-1)}{2 h} \pi, \quad j, m=1,2, \cdots,
\end{aligned}
$$

and

$$
D_{m}^{1}-D_{m}^{2}=-\frac{2}{h} \sum_{j=1} C_{j}\left[\begin{array}{l}
\frac{\sin k_{j} c}{\left(k_{m}^{*}\right)^{2}-\left(k_{j}\right)^{2}} \text { for } k_{j} \neq k_{m}^{*}, \\
\frac{1}{2\left(k_{j}\right)^{2}}\left(k_{j} h \cdot \cos k_{j} c-\sin k_{j} c\right) \text { for } k_{j}=k_{m}^{*}, \\
j, m=1,2, \cdots .
\end{array}\right.
$$

Equations (17-21) result from comparison of the potential functions at common boundaries. These equations ensure equal pressure at the boundaries formed by neighbouring fluid domains. With the relations derived, the description of the problem has been reduced to fewer unknown constants, i.e. to $B_{j}$ and $C_{j}(j=1,2, \cdots)$. Obviously, the fluid velocities at the common boundaries of matching domains must be the same. To this end, not only the fluid pressure, but also the normal components of the velocity field at the common boundaries should be uniquely defined. This condition makes it possible to express all constants in terms of the constants $\dot{A}_{n}$ that enter the description of the plate deflection. Thus, with respect to the above, it is necessary to calculate the horizontal velocity components. For the fluid below the plate $(-b \leq x \leq+b, 0 \leq z \leq d)$, one obtains

$$
\begin{aligned}
u= & \frac{\partial \varphi}{\partial x}=\sum_{n=1} \dot{A}_{n} \frac{\cosh r_{n} z}{\sinh r_{n} d} \cos r_{n}(x+b)+ \\
& +\sum_{m=1} k_{m}\left(E_{m}^{1} \frac{\sinh k_{m} x}{\cosh k_{m} b}+E_{m}^{2} \frac{\cosh k_{m} x}{\sinh k_{m} b}\right) \cos k_{m} z, \quad r_{n}=\frac{n \pi}{2 b} .
\end{aligned}
$$

For the upper fluid domain $(-b \leq x \leq+b, 0 \leq z \leq h)$, we have a similar relation:

$$
\begin{aligned}
u= & \frac{\partial \phi}{\partial x}=-\sum_{n=1} \dot{A}_{n} \frac{1}{v_{n}}\left[\exp \left(-r_{n} z\right)-\exp r_{n}(z-2 h)\right] \cos r_{n}(x+b)+ \\
& +\sum_{m=1} k_{m}^{*}\left(D_{m}^{1} \frac{\sinh k_{m}^{*} x}{\cosh k_{m}^{*} b}+D_{m}^{2} \frac{\cosh k_{m}^{*} x}{\sinh k_{m}^{*} b}\right) \cos k_{m} z .
\end{aligned}
$$

It should be stressed that the vertical coordinate $z$ in equation (23) differs from that in equation (22) (they are local with respect to corresponding fluid domains). With equations (22) and (23) it is possible to calculate horizontal velocities at the boundaries $x= \pm b$. For the right boundary $(x=+b)$, equations (22) and (23) give 


$$
\begin{aligned}
& 0 \leq z \leq d \\
& u=\left.\frac{\partial \varphi}{\partial x}\right|_{x=b}= \\
& =\sum_{n=1} \dot{A}_{n}(-1)^{n} \frac{\cosh r_{n} z}{\sinh r_{n} d}+\sum_{m=1} k_{m}\left(E_{m}^{1} \tanh k_{m} b+E_{m}^{2} \frac{1}{\tanh k_{m} b}\right) \cos k_{m} z= \\
& =\sum_{j} B_{j} \cos k_{j} z
\end{aligned}
$$

and

$$
\begin{aligned}
c \leq z \leq H & \\
u=\left.\frac{\partial \phi}{\partial x}\right|_{x=b}= & -\sum_{n=1} \dot{A}_{n}(-1)^{n} \frac{1}{v_{n}}\left[\exp -r_{n}(z-c)-\exp -r_{n}(z-c-2 h)\right]+ \\
& +\sum_{m=1} k_{m}^{*}\left(D_{m}^{1} \tanh k_{m}^{*} b+D_{m}^{2} \frac{1}{\tanh k_{m}^{*} b}\right) \cos k_{m}^{*}(z-c)= \\
= & \sum_{j} B_{j} \cos k_{j} z .
\end{aligned}
$$

In a similar way, for the boundary at $(x=-b)$, the following relations hold:

$$
\begin{aligned}
& 0 \leq z \leq d \\
& u=\left.\frac{\partial \varphi}{\partial x}\right|_{x=b}= \\
& \sum_{n=1} \dot{A}_{n} \frac{\cosh r_{n} z}{\sinh r_{n} d}+\sum_{m=1} k_{m}\left(-E_{m}^{1} \tanh k_{m} b+E_{m}^{2} \frac{1}{\tanh k_{m} b}\right) \cos k_{m} z= \\
& \quad=-\sum_{j} C_{j} \cos k_{j} z,
\end{aligned}
$$

and

$$
\begin{aligned}
c \leq z \leq H & \\
u=\left.\frac{\partial \phi}{\partial x}\right|_{x=b}= & -\sum_{n=1} \dot{A}_{n} \frac{1}{v_{n}}\left[\exp -r_{n}(z-c)-\exp -r_{n}(z-c-2 h)\right]+ \\
& +\sum_{m=1} k_{m}^{*}\left(-D_{m}^{1} \tanh k_{m}^{*} b+D_{m}^{2} \frac{1}{\tanh k_{m}^{*} b}\right) \cos k_{m}^{*}(z-c)= \\
= & -\sum_{j} C_{j} \cos k_{j} z .
\end{aligned}
$$

The difference $(c-d)$ in the relations equals the plate thickness. Knowing that the constants $E_{m}^{1}, E_{m}^{2}$, and $D_{n}^{1}, D_{n}^{1}(m, n=1,2, \cdots)$ in the above relations depend on $B_{j}$ and $C_{j}(j=1,2, \cdots)$, one can use equations (22-27) to express the latter constants 
in terms of $\dot{A}_{n}(n=1,2, \cdots)$. In order to find the desired relations, equations (24-27) are multiplied in succession by $\cos k_{j} z(j=1,2, \cdots)$ and then integrated within the range $(0 \leq z \leq H)$. Such a procedure leads to two systems of equations:

$$
\begin{aligned}
& x=b \\
& \sum_{n=1} \dot{A}_{n}(-1)^{n} \frac{1}{\sinh r_{n} d} \int_{0}^{d} \cosh r_{n} z \cdot \cos k_{j} z d z+ \\
& \quad+\sum_{m=1} k_{m}\left[E_{m}^{1} \tanh k_{m} b+E_{m}^{2} \frac{1}{\tanh k_{m} b}\right] \int_{0}^{d} \cos k_{m} z \cdot \cos k_{j} z d z+ \\
& -\sum_{n=1} \dot{A}_{n}(-1)^{n} \frac{1}{v_{n}} \int_{c}^{H}\left[e^{-r_{n}(z-c)}-e^{-r_{n}(z-c-2 h)}\right] \cdot \cos k_{j} z d z+ \\
& \quad+\sum_{m=1} k_{m}^{*}\left[D_{m}^{1} \tanh k_{m}^{*} b+D_{m}^{2} \frac{1}{\tanh k_{m}^{*} b}\right] \int_{c}^{H} \cos k_{m}^{*}(z-c) \cdot \cos k_{j} z d z= \\
& \quad=B_{j} \frac{H}{2},
\end{aligned}
$$

and

$$
\begin{aligned}
& x=-b \\
& \sum_{n=1} \dot{A}_{n} \frac{1}{\sinh r_{n} d} \int_{0}^{d} \cosh r_{n} z \cdot \cos k_{j} z d z+ \\
& \quad+\sum_{m=1} k_{m}\left[-E_{m}^{1} \tanh k_{m} b+E_{m}^{2} \frac{1}{\tanh k_{m} b}\right] \int_{0}^{d} \cos k_{m} z \cdot \cos k_{j} z d z+ \\
& \quad-\sum_{n=1} \dot{A}_{n} \frac{1}{v_{n}} \int_{c}^{H}\left[e^{-r_{n}(z-c)}-e^{-r_{n}(z-c-2 h)}\right] \cdot \cos k_{j} z d z+ \\
& \quad+\sum_{m=1} k_{m}^{*}\left[-D_{m}^{1} \tanh k_{m}^{*} b+D_{m}^{2} \frac{1}{\tanh k_{m}^{*} b}\right] \int_{c}^{H} \cos k_{m}^{*}(z-c) \cdot \cos k_{j} z d z= \\
& \quad=-C_{j} \frac{H}{2} .
\end{aligned}
$$

The formulae written above have a complicated structure. In order to make our further discussion clear and to simplify the description of the problem, we confine our attention to a finite number of terms in the infinite series entering all the above relations. Thus, let us assume that $n a$ denotes the number of constants $\dot{A}_{n}$ taken into account. And, similarly, $n d$, ne and $n j$ denote the numbers of constants $D_{m}, E_{m}$ and $B_{j}\left(C_{j}\right)$, 
respectively. With respect to these finite numbers of terms in the series, it is convenient to make the following substitutions:

$$
\begin{aligned}
J A_{j}^{n} & =\frac{2}{H}(-1)^{n}\left[\frac{1}{\sinh r_{n} d} \int_{0}^{d} \cosh r_{n} z \cdot \cos k_{j} z d z+\right. \\
& \left.-\frac{1}{v_{n}} \int_{c}^{H}\left(e^{-r_{n}(z-c)}-e^{-r_{n}(z-c-2 h)}\right) \cdot \cos k_{j} z d z\right], \\
J D_{j}^{m} & =\frac{2}{H} k_{m}^{*} \tanh k_{m}^{*} b \int_{c}^{H} \cos k_{m}^{*}(z-c) \cdot \cos k_{j} z d z, \\
J E_{j}^{m} & =\frac{2}{H} k_{m} \tanh k_{m} b \int_{0}^{d} \cos k_{m} z \cdot \cos k_{j} z d z,
\end{aligned}
$$

and

$$
\begin{aligned}
& K A_{j}^{n}=\frac{2}{H} \frac{1}{\sinh r_{n} d} \int_{0}^{d} \cosh r_{n} z \cdot \cos k_{j} z d z+ \\
& -\frac{1}{v_{n}} \int_{c}^{H}\left[e^{-r_{n}(z-c)}-e^{-r_{n}(z-c-2 h)}\right] \cdot \cos k_{j} z d z, \\
& K D_{j}^{m}=\frac{2}{H} k_{m}^{*} \frac{1}{\tanh k_{m}^{*} b} \int_{c}^{H} \cos k_{m}^{*}(z-c) \cdot \cos k_{j} z d z, \\
& K E_{j}^{m}=\frac{2}{H} k_{m} \frac{1}{\tanh k_{m} b} \int_{0}^{d} \cos k_{m} z \cdot \cos k_{j} z d z .
\end{aligned}
$$

From substitution of (30) and (31) into relations (28) and (29), we obtain

$$
\begin{aligned}
& x=b \\
& \sum_{n=1}^{n a} \dot{A}_{n} J A_{j}^{n}+\sum_{m=1}^{n e}\left(E_{m}^{1} J E_{j}^{m}+E_{m}^{2} K E_{j}^{m}\right)+\sum_{m=1}^{n d}\left(D_{m}^{1} J D_{j}^{m}+D_{m}^{2} K D_{j}^{m}\right)=B_{j},
\end{aligned}
$$

and

$$
\begin{aligned}
& x=-b \\
& \sum_{n=1}^{n a} \dot{A}_{n} K A_{j}^{n}+\sum_{m=1}^{n e}\left(-E_{m}^{1} J E_{j}^{m}+E_{m}^{2} K E_{j}^{m}\right)+ \\
& +\sum_{m=1}^{n d}\left(-D_{m}^{1} J D_{j}^{m}+D_{m}^{2} K D_{j}^{m}\right)=-C_{j} .
\end{aligned}
$$


Equations (32) and (33) are written for $j=1,2, \cdots, n j$. The system of equations obtained in this way may be written in a more compact, matrix form:

$$
\begin{aligned}
& (\boldsymbol{B})=[\boldsymbol{J A}](\dot{\boldsymbol{A}})+[\boldsymbol{J E}](\boldsymbol{E} 1)+[\boldsymbol{K E}](\boldsymbol{E} 2)+[\boldsymbol{J D}](\boldsymbol{D} 1)+[\boldsymbol{K D}](\boldsymbol{D} 2), \\
& (\boldsymbol{C})=-\{[\boldsymbol{K A}](\dot{\boldsymbol{A}})-[\boldsymbol{J E}](\boldsymbol{E} 1)+[\boldsymbol{K} \boldsymbol{E}](\boldsymbol{E} 2)-[\boldsymbol{J} \boldsymbol{D}](\boldsymbol{D} 1)+[\boldsymbol{K} \boldsymbol{D}](\boldsymbol{D} 2)\} .
\end{aligned}
$$

The vector matrices $(\boldsymbol{B})$ and $(\boldsymbol{C})$ in these equations have $n j$ elements. The dimensions of the square matrices correspond to the number of terms taken into account in the associated series. With the notation presented above, the dimensions of the associated matrices are

$$
\boldsymbol{J} \boldsymbol{A}, \boldsymbol{K} \boldsymbol{A} \rightarrow(n j \times n a), \boldsymbol{J} \boldsymbol{E}, \boldsymbol{K} \boldsymbol{E} \rightarrow(n j \times n e), \boldsymbol{J} \boldsymbol{D}, \boldsymbol{K} \boldsymbol{D} \rightarrow(n j \times n d) .
$$

In accordance with the finite matrix description, equations (17-21) are also replaced by a finite system of equations with a finite number of terms. These equations are written in the following matrix form:

$$
\begin{aligned}
& E_{0}=[\boldsymbol{E} \boldsymbol{O B}](\boldsymbol{B}+\boldsymbol{C}), \\
& (\boldsymbol{E} 1+\boldsymbol{E} 2)=[\boldsymbol{E} \boldsymbol{B}](\boldsymbol{B}), \\
& (\boldsymbol{E} 1-\boldsymbol{E} 2)=[\boldsymbol{E} \boldsymbol{B}](\boldsymbol{C}), \\
& (\boldsymbol{D} 1+\boldsymbol{D} 2)=[\boldsymbol{D B}](\boldsymbol{B}), \\
& (\boldsymbol{D} 1-\boldsymbol{D} 2)=[\boldsymbol{D B}](\boldsymbol{C}),
\end{aligned}
$$

where $(1 \times n j)$ matrix $\boldsymbol{E} \boldsymbol{O B}$ reads

$$
\boldsymbol{E O} \boldsymbol{B}=\left[\ldots \ldots,-\frac{1}{2 d} \frac{1}{\left(k_{j}\right)^{2}} \sin k_{j} d, \ldots \ldots \ldots . .\right.
$$

At the same time, equations (18) and (19) lead to the $(n e \times n j)$ matrix $\boldsymbol{E} \boldsymbol{B}$ :

$$
\boldsymbol{E} \boldsymbol{B}_{m, j}=-\frac{2}{d}\left\{\begin{array}{l}
\frac{(-1)^{m} \sin k_{j} d}{\left(k_{j}\right)^{2}-\left(k_{m}\right)^{2}} \text { for } k_{j} \neq k_{m} \\
\frac{d}{2 k_{j}} \quad \text { for } k_{j}=k_{m} .
\end{array}\right.
$$

Finally, the $(n d \times n j)$ matrix $\boldsymbol{D B}$ is defined by the formula

$$
\boldsymbol{D} \boldsymbol{B}_{m, j}=-\frac{2}{h}\left\{\begin{array}{l}
\frac{\sin k_{j} c}{\left(k_{m}^{*}\right)^{2}-\left(k_{j}\right)^{2}} \text { for } k_{j} \neq k_{m}^{*} \\
\frac{1}{2\left(k_{j}\right)^{2}}\left(k_{j} h \cdot \cos k_{j} c-\sin k_{j} c\right) \quad \text { for } k_{j}=k_{m}^{*} .
\end{array}\right.
$$

The sum and difference of the two equations (34) lead to the following relations

$$
\begin{aligned}
& (\boldsymbol{X})=(\boldsymbol{B}+\boldsymbol{C})=[\boldsymbol{J} \boldsymbol{A}-\boldsymbol{K} \boldsymbol{A}](\dot{\boldsymbol{A}})+2[\boldsymbol{J E}](\boldsymbol{E} 1)+2[\boldsymbol{J} \boldsymbol{D}](\boldsymbol{D} 1), \\
& (\boldsymbol{Y})=(\boldsymbol{B}-\boldsymbol{C})=[\boldsymbol{J} \boldsymbol{A}+\boldsymbol{K} \boldsymbol{A}](\dot{\boldsymbol{A}})+2[\boldsymbol{K E}](\boldsymbol{E} 2)+2[\boldsymbol{K} \boldsymbol{D}](\boldsymbol{D} 2) .
\end{aligned}
$$


On the other hand, equations (36) give

$$
\begin{aligned}
& 2(\boldsymbol{E} 1)=[\boldsymbol{E} \boldsymbol{B}](\boldsymbol{B}+\boldsymbol{C})=[\boldsymbol{E} \boldsymbol{B}](\boldsymbol{X}), \\
& 2(\boldsymbol{E} 2)=[\boldsymbol{E B}](\boldsymbol{B}-\boldsymbol{C})=[\boldsymbol{E} \boldsymbol{B}](\boldsymbol{Y}), \\
& 2(\boldsymbol{D} 1)=[\boldsymbol{D B}](\boldsymbol{B}+\boldsymbol{C})=[\boldsymbol{D} \boldsymbol{B}](\boldsymbol{X}), \\
& 2(\boldsymbol{D} 2)=[\boldsymbol{D B}](\boldsymbol{B}-\boldsymbol{C})=[\boldsymbol{D B}](\boldsymbol{Y}) .
\end{aligned}
$$

From substitution of equations (41) into relations (40), the following system of equations is obtained:

$$
\begin{aligned}
& {[\boldsymbol{R A}](\boldsymbol{X})=[\boldsymbol{J} \boldsymbol{A}-\boldsymbol{K} \boldsymbol{A}](\dot{\boldsymbol{A}}),} \\
& {[\boldsymbol{R B}](\boldsymbol{Y})=[\boldsymbol{J} \boldsymbol{A}+\boldsymbol{K} \boldsymbol{A}](\dot{\boldsymbol{A}}),}
\end{aligned}
$$

where

$$
\begin{aligned}
& {[R A]=[I]-[J E][E B]-[J D][D B],} \\
& {[R B]=[I]-[K E][E B]-[K D][D B] .}
\end{aligned}
$$

The matrix $[\boldsymbol{I}]$ in these relations is the $(n j \times n j)$ unit diagonal matrix. From equations (44), the following solutions are obtained:

$$
\begin{aligned}
& (\boldsymbol{X})=[\boldsymbol{R A}]^{-1}[\boldsymbol{J A}-\boldsymbol{K A}](\dot{A})=[\boldsymbol{X} \boldsymbol{A}](\dot{\boldsymbol{A}}), \\
& (\boldsymbol{Y})=[\boldsymbol{R} \boldsymbol{B}]^{-1}[\boldsymbol{J} \boldsymbol{A}+\boldsymbol{K} \boldsymbol{A}](\dot{\boldsymbol{A}})=[\boldsymbol{Y} \boldsymbol{A}](\dot{\boldsymbol{A}}) .
\end{aligned}
$$

Substitution of these relations into equations (36) and (41) gives

$$
\begin{aligned}
& E_{0}=[\boldsymbol{E O B}][\boldsymbol{X A}](\dot{A}) \\
& (\boldsymbol{E} 1)=\frac{1}{2}[\boldsymbol{E B}][\boldsymbol{X A}](\dot{A}),(\boldsymbol{E} 2)=\frac{1}{2}[\boldsymbol{E B}][\boldsymbol{Y A}](\dot{A}), \\
& (\boldsymbol{D} 1)=\frac{1}{2}[\boldsymbol{D B}][\boldsymbol{X A}](\dot{A}),(\boldsymbol{D} 2)=\frac{1}{2}[\boldsymbol{D B}][\boldsymbol{Y} \boldsymbol{A}](\dot{\boldsymbol{A}}) .
\end{aligned}
$$

With these relations, all unknown constants (parameters) of the problem considered are expressed in terms of independent parameters that correspond to the plate deflection. It is important to note that none of the matrices in these equations, i.e. $[\boldsymbol{E O B}]$, $[\boldsymbol{E} \boldsymbol{B}],[\boldsymbol{D B}],[\boldsymbol{X} \boldsymbol{A}]$ or $[\boldsymbol{Y} \boldsymbol{A}]$, depends on time.

\section{Free Vibrations of the Plate and Co-vibrating Mass of Fluid}

The solution presented in the preceding section corresponds to a general case of standing waves (the pressure at the surface $z=H$ is zero) and arbitrary motion of the plate. In order to find a fundamental set of natural frequencies of the band plate submerged 
in a layer of fluid, both the deflection of the plate and potential functions are expressed in the following forms:

$$
\begin{aligned}
& w(x, t)=W(x) \exp (i \omega t), \\
& \phi^{*}(x, z, t)=i \omega \phi(x, z) \exp (i \omega t), \\
& \varphi^{*}(x, z, t)=i \omega \varphi(x, z) \exp (i \omega t) .
\end{aligned}
$$

Substitution of these relations into equation (1) gives the fundamental equation for the free vibrations of the plate submerged in fluid:

$$
-m_{p l} \omega^{2}\left[W(x)+\frac{\rho}{m_{p l}}(\varphi-\phi)_{p l}\right]+D^{*} \frac{\partial^{4} W(x)}{\partial x^{4}}=0 .
$$

The frequency $\omega$ in this relation is different from that corresponding to free vibrations of the plate in air. The potential functions $\phi$ and $\varphi$ in (47) are described by the general formulae given in the previous section. Vibrations of the plate in fluid are accompanied by the so-called co-vibrating mass of fluid, which leads to the above-mentioned differences in natural frequencies. This co-vibrating mass of fluid leads to a shift of the natural frequencies of the coupled vibrations to smaller values as compared to frequencies in air. At the same time, as in the case of vibrations in air, the plate deflection and its space derivatives are described by the formulae

$$
\begin{aligned}
& W(x)=\sum_{1}^{n a} A_{n} \sin r_{n}(x+b), \\
& \frac{\partial^{4} W(x)}{\partial x^{4}}=\sum_{1}^{n a} A_{n}\left(r_{n}\right)^{4} \sin r_{n}(x+b), \quad r_{n}=\frac{n \pi}{2 b} .
\end{aligned}
$$

From substitution of these relations into equation (47), one obtains

$$
\begin{gathered}
-m_{p l} \omega^{2} \sum_{n=1}^{N A} A_{n} \sin r_{n}(x+b)+ \\
+D^{*} \sum_{n=1}^{N A} A_{n}\left(r_{n}\right)^{4} \sin r_{n}(x+b)-m_{p l} \omega^{2} \frac{\rho}{m_{p l}}(\varphi-\phi)_{p l}=0 .
\end{gathered}
$$

Multiplication of this equation in succession by $\sin r_{n}(x+b)(n=1,2, \cdots, n j)$ and integration of the result within the range $(-b \leq x \leq+b)$, leads to the system of equations:

$$
\begin{aligned}
& \frac{b}{\left(r_{n}\right)^{4}} A_{n}+\frac{1}{\left(r_{n}\right)^{4}} \frac{\rho}{m_{p l}} \int_{-b}^{+b}(\varphi-\phi)_{p l} \sin r_{n}(x+b) d x-\frac{D^{*} b}{m_{p l} \omega^{2}} A_{n}=0, \\
& n=1,2, \cdots
\end{aligned}
$$


The term $\rho(\varphi-\phi)_{p l}$ in equation (49) corresponds to the mass of fluid vibrating together with the plate. From equations (31) and (32), it follows that

$$
\begin{aligned}
(\varphi-\phi)_{p l} & =\sum_{n=1}^{N A} A_{n} \frac{1}{r_{n}}\left(\frac{1}{\tanh r_{n} d}+\frac{1-e^{-2 r_{n} h}}{1+e^{-2 r_{n} h}}\right) \sin r_{n}(x+b)+ \\
& +E_{0}+\sum_{m=1}^{N E}(-1)^{m}\left(E_{m}^{1} \frac{\cosh k_{m} x}{\cosh k_{m} b}+E_{m}^{2} \frac{\sinh k_{m} x}{\sinh k_{m} b}\right)+ \\
& -\sum_{m=1}^{N D}\left(D_{m}^{1} \frac{\cosh k_{m}^{*} x}{\cosh k_{m}^{*} b}+D_{m}^{2} \frac{\sinh k_{m}^{*} x}{\sinh k_{m}^{*} b}\right), \\
r_{n}=\frac{n \pi}{2 b}, & k_{m}=\frac{m \pi}{d}, \quad k_{m}^{*}=\frac{2 m-1}{2 h} \pi .
\end{aligned}
$$

Substitution of this equation into (50) gives

$$
\begin{aligned}
& \frac{b}{\left(r_{n}\right)^{4}}\left[1+\frac{\rho}{m_{p l}} \frac{1}{r_{n}}\left(\frac{1}{\tanh r_{n} d}+\frac{1-e^{-2 r_{n} h}}{1+e^{-2 r_{n} h}}\right)\right] A_{n}-\frac{D^{*} b}{m_{p l} \omega^{2}} A_{n}+ \\
& +\frac{\rho}{m_{p l}\left(r_{n}\right)^{4}}\left[E_{0} \int_{-b}^{+b} \sin r_{n}(x+b) d x+\right. \\
& +\sum_{m=1}^{N E}(-1)^{m} \int_{-b}^{+b}\left(E_{m}^{1} \frac{\cosh k_{m} x}{\cosh k_{m} b}+E_{m}^{2} \frac{\sinh k_{m} x}{\sinh k_{m} b}\right) \sin r_{n}(x+b) d x+ \\
& \left.-\sum_{m=1}^{N D} \int_{-b}^{+b}\left(D_{m}^{1} \frac{\cosh k_{m}^{*} x}{\cosh k_{m}^{*} b}+D_{m}^{2} \frac{\sinh k_{m}^{*} x}{\sinh k_{m}^{*} b}\right) \sin r_{n}(x+b) d x\right]=0, n=1,2, \cdots
\end{aligned}
$$

Obviously, all constants $E$ and $D$ in these equations depend on $A_{n}(n=1,2, \cdots)$, and therefore, the final system of equations will uniquely depend on these latter constants. From these equations it follows that, in the limit $\rho \rightarrow 0$, we arrive at equations corresponding to free vibration of the plate in air. The integrals in equations (52) are expressed in exact form as 


$$
\begin{aligned}
& L A_{n}^{0}=\int_{-b}^{+b} \sin r_{n}(x+b) d x=\frac{1}{r_{n}}\left[1-(-1)^{n}\right], \\
& L A_{n}^{m}=\frac{(-1)^{m}}{\cosh k_{m} b} \int_{-b}^{+b} \cosh k_{m} x \cdot \sin r_{n}(x+b) d x= \\
& =\frac{(-1)^{m} r_{n}}{\left(k_{m}\right)^{2}+\left(r_{n}\right)^{2}}\left[1-(-1)^{n}\right] \text {, } \\
& L B_{n}^{m}=\frac{(-1)^{m}}{\sinh k_{m} b} \int_{-b}^{+b} \sinh k_{m} x \cdot \sin r_{n}(x+b) d x= \\
& -\frac{(-1)^{m} r_{n}}{\left(k_{m}\right)^{2}+\left(r_{n}\right)^{2}}\left[1+(-1)^{n}\right] \\
& K A_{n}^{m}=\frac{1}{\cosh k_{m}^{*} b} \int_{-b}^{+b} \cosh k_{m}^{*} x \cdot \sin r_{n}(x+b) d x= \\
& \frac{r_{n}}{\left(k_{m}^{*}\right)^{2}+\left(r_{n}\right)^{2}}\left[1-(-1)^{n}\right] \text {, } \\
& K B_{n}^{m}=\frac{1}{\sinh k_{m}^{*} b} \int_{-b}^{+b} \sinh k_{m}^{*} x \cdot \sin r_{n}(x+b) d x= \\
& -\frac{r_{n}}{\left(k_{m}^{*}\right)^{2}+\left(r_{n}\right)^{2}}\left[1+(-1)^{n}\right] \text {. }
\end{aligned}
$$

With these results, equations (52) may be written in the following form:

$$
\begin{aligned}
\frac{b}{\left(r_{n}\right)^{4}}[1+ & \left.\frac{\rho}{m_{p l}} \frac{1}{r_{n}}\left(\frac{1}{\tanh r_{n} d}+\frac{1-e^{-2 r_{n} h}}{1+e^{-2 r_{n} h}}\right)\right] A_{n}-\frac{D^{*} b}{m_{p l} \omega^{2}} A_{n}+ \\
& +\frac{\rho}{m_{p l}\left(r_{n}\right)^{4}}\left[L A_{n}^{0} E_{0}+\sum_{m=1}^{N E}\left(L A_{n}^{m} E_{m}^{1}+L B_{n}^{m} E_{m}^{2}\right)+\right. \\
& \left.-\sum_{m=1}^{N D}\left(K A_{n}^{m} D_{m}^{1}+K B_{n}^{m} D_{m}^{2}\right)\right]=0, n=1,2, \cdots
\end{aligned}
$$

Finally, all these equations are written in the matrix form

$$
\begin{gathered}
{[\boldsymbol{A B}](\boldsymbol{A})+(\boldsymbol{E O}) E_{0}+[\boldsymbol{L A}](\boldsymbol{E} \mathbf{1})+} \\
{[\boldsymbol{L B}](\boldsymbol{E 2})-[\boldsymbol{K A}](\boldsymbol{D} \mathbf{1})-[\boldsymbol{K B}](\boldsymbol{E} \mathbf{2})-\lambda[\boldsymbol{I}](\boldsymbol{A})=\mathbf{0},}
\end{gathered}
$$

where

$$
\lambda=\frac{b D^{*}}{m_{p l} \omega^{2}} .
$$


The elements of the diagonal matrix $[\boldsymbol{A B}]$ and vector matrix $(\boldsymbol{E} \mathbf{0})$ are described by the formulae

$$
\begin{aligned}
A B_{n}^{n} & =\frac{b}{\left(r_{n}\right)^{4}}\left[1+\frac{\rho}{m_{p l}} \frac{1}{r_{n}}\left(\frac{1}{\tanh r_{n} d}+\frac{1-e^{-2 r_{n} h}}{1+e^{-2 r_{n} h}}\right)\right], \\
E O_{n} & =\frac{\rho}{m_{p l}\left(r_{n}\right)^{4}} \frac{1-(-1)^{n}}{r_{n}} .
\end{aligned}
$$

Substituting equations (57), (53) and (45) into (55) and making simple manipulations, one obtains the final system of equations

$$
([\boldsymbol{A A}]-\lambda[\boldsymbol{I}])(\boldsymbol{A})=\mathbf{0} .
$$

The matrix $[\boldsymbol{A A}]$ in this equation is a square matrix. With this matrix, dependent on the plate parameters and a gap between the plate and the fluid bottom, it is possible

\begin{tabular}{|c|c|c|c|c|c|}
\hline & \multicolumn{5}{|c|}{ Eigenfrequencies of the plate in air } \\
\hline & $\omega_{1}$ & $\omega_{2}$ & $\omega_{3}$ & $\omega_{4}$ & $\omega_{5}$ \\
\hline & 61.201 & 244.803 & 550.807 & 979.212 & 1530.019 \\
\hline \multirow{2}{*}{$\begin{array}{c}\mathrm{c} \\
{[\mathrm{cm}]}\end{array}$} & \multicolumn{5}{|c|}{ Eigenfrequencies of the plate in water } \\
\hline & $\omega_{1}$ & $\omega_{2}$ & $\omega_{3}$ & $\omega_{4}$ & $\omega_{5}$ \\
\hline 1 & 2.510 & 20.309 & 66.427 & 158.082 & 299.403 \\
\hline 2 & 3.946 & 32.074 & 101.374 & 239.614 & 440.465 \\
\hline 3 & 4.870 & 39.564 & 121.811 & 285.119 & 513.626 \\
\hline 4 & 5.567 & 45.081 & 135.855 & 314.741 & 558.640 \\
\hline 5 & 6.127 & 49.385 & 146.166 & 335.256 & 588.357 \\
\hline 6 & 6.595 & 52.849 & 154.023 & 349.959 & 608.760 \\
\hline 7 & 6.995 & 55.694 & 160.154 & 360.725 & 623.125 \\
\hline 8 & 7.343 & 58.063 & 165.021 & 368.724 & 633.413 \\
\hline 9 & 7.650 & 60.056 & 168.933 & 374.727 & 640.876 \\
\hline 10 & 7.924 & 61.746 & 172.108 & 379.263 & 646.343 \\
\hline 11 & 8.170 & 63.190 & 174.706 & 382.706 & 650.381 \\
\hline 12 & 8.393 & 64.430 & 176.845 & 385.327 & 653.383 \\
\hline 13 & 8.596 & 65.500 & 178.616 & 387.325 & 655.629 \\
\hline 14 & 8.782 & 66.427 & 180.090 & 388.848 & 657.320 \\
\hline 15 & 8.954 & 67.235 & 181.323 & 390.005 & 658.600 \\
\hline 16 & 9.114 & 67.941 & 182.359 & 390.882 & 659.575 \\
\hline 17 & 9.263 & 68.561 & 183.232 & 391.543 & 660.323 \\
\hline 18 & 9.402 & 69.107 & 183.972 & 392.037 & 660.899 \\
\hline 19 & 9.532 & 69.592 & 184.601 & 392.401 & 661.347 \\
\hline 20 & 9.656 & 70.023 & 185.140 & 392.667 & 661.698 \\
\hline
\end{tabular}
to calculate the associated set of eigenfrequencies of the plate.

Table 1. Steel plate, $\delta=4 \mathrm{~mm}, D=1207.38 \mathrm{~kg} \cdot \mathrm{m}^{3} \cdot \mathrm{s}^{-2}, H=0.60 \mathrm{~m}$

The solution of the problem presented above is illustrated by numerical examples below. Two plates are considered. The first one is a steel plate of thickness $\delta=4 \mathrm{~mm}$, and the second one of thickness $\delta=20 \mathrm{~mm}$ is made of reinforced concrete. In order 
to investigate the influence of the gap between the plates and the fluid bottom on the eigenfrequencies of these plates, a chosen set of the gap widths is taken into account. Some of the results obtained in numerical calculations are drawn up in tables 1 and 2.

Table 2. Reinforced concrete plate, $\delta=2 \mathrm{~mm}, D=2.8964 \cdot 10^{4} \mathrm{~kg} \cdot \mathrm{m}^{3} \cdot \mathrm{s}^{-2}, H=0.60 \mathrm{~m}$

\begin{tabular}{|c|c|c|c|c|c|}
\hline \multirow{2}{*}{} & \multicolumn{5}{|c|}{ Eigenfrequencies of the plate in air } \\
\cline { 2 - 6 } & $\omega_{1}$ & $\omega_{2}$ & $\omega_{3}$ & $\omega_{4}$ & $\omega_{5}$ \\
\cline { 2 - 6 } & 237.546 & 950.184 & 2137.915 & 3800.739 & 5938.655 \\
\hline $\mathrm{c}$ \\
{$[\mathrm{cm}]$} & $\omega_{1}$ & $\omega_{2}$ & $\omega_{3}$ & $\omega_{4}$ & $\omega_{5}$ \\
\hline 3 & 14.838 & 121.016 & 387.919 & 925.757 & 1722.011 \\
\hline 4 & 20.212 & 165.480 & 513.935 & 1217.468 & 2207.365 \\
\hline 5 & 23.972 & 196.111 & 594.404 & 1394.321 & 2484.107 \\
\hline 6 & 26.896 & 219.299 & 651.606 & 1512.898 & 2660.854 \\
\hline 7 & 29.288 & 237.646 & 694.424 & 1596.309 & 2780.070 \\
\hline 8 & 31.307 & 252.544 & 727.481 & 1656.660 & 2863.118 \\
\hline 9 & 33.049 & 264.851 & 753.535 & 1701.131 & 2922.216 \\
\hline 10 & 34.575 & 275.147 & 774.379 & 1734.308 & 2964.911 \\
\hline 11 & 35.928 & 283.841 & 791.243 & 1759.273 & 2996.108 \\
\hline 12 & 37.140 & 291.240 & 805.008 & 1778.167 & 3019.111 \\
\hline 13 & 38.236 & 297.575 & 816.328 & 1792.520 & 3036.201 \\
\hline 14 & 39.233 & 303.031 & 825.693 & 1803.443 & 3048.985 \\
\hline 15 & 40.146 & 307.752 & 833.484 & 1811.758 & 3058.608 \\
\hline 16 & 40.988 & 311.857 & 839.996 & 1818.079 & 3065.895 \\
\hline 17 & 41.767 & 315.441 & 845.466 & 1822.870 & 3071.447 \\
\hline 18 & 42.493 & 318.585 & 850.081 & 1826.484 & 3075.705 \\
\hline 19 & 43.172 & 321.357 & 853.992 & 1829.190 & 3078.992 \\
\hline 20 & 43.810 & 323.812 & 857.323 & 1831.196 & 3081.550 \\
\hline
\end{tabular}

Table 1 contains a set of five lowest eigenfrequencies of the steel plate. Similar results for the reinforced concrete plate are shown in table 2 . In order to obtain a better insight into the solutions obtained, the lowest eigenfrequencies are illustrated in Fig. 2. From the data collected in these tables and from the plots in this figure, it may be

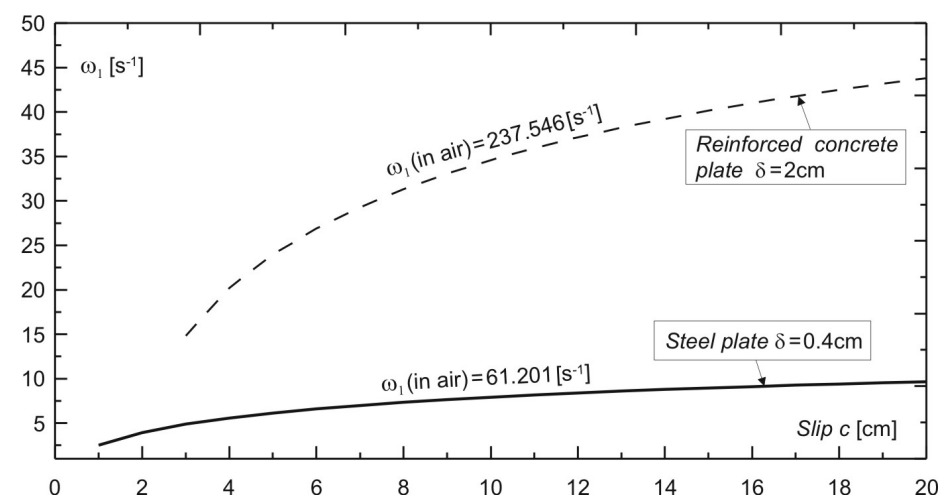

Fig. 2. Eigenfrequencies of steel and reinforced plates versus slip width 
seen that the lowest frequencies correspond to the lowest gaps. It is important for practical reasons that the formulation presented above makes it possible to calculate the smallest plate eigenfrequency for a given distance between the horizontal plate and the fluid (sea) bottom.

\section{Flow-induced Vibrations of the Plate}

Results of the previous sections are employed here to investigate forced vibrations of the horizontal plate induced by gravitational waves. In the case considered, the plate is submerged in a semi-infinite layer of fluid, as shown schematically in Fig. 3.

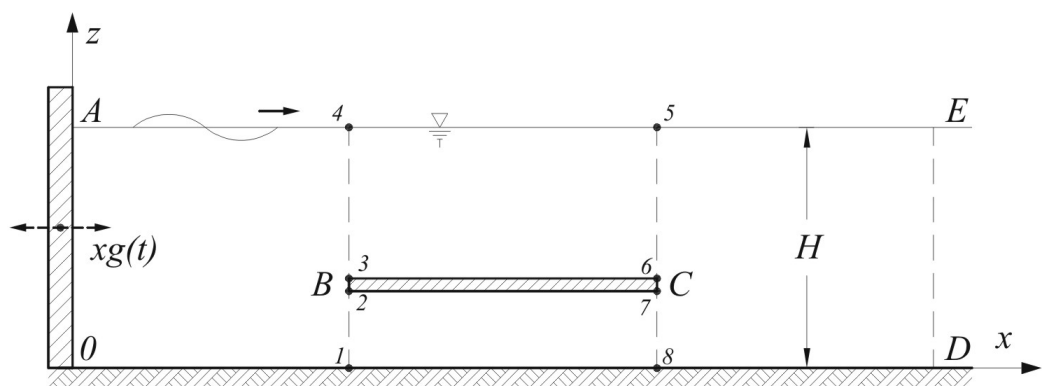

Fig. 3. Simply supported elastic plate $B C$ submerged in a semi-infinite layer of fluid

Water waves are generated by a piston-type generator (rigid vertical wall $O A$ ) placed at the beginning of the layer. The generator motion is described by the formula

$$
x g(t)=x g \exp (i \omega t)
$$

where $x g$ is the generation amplitude.

The potential function for the fluid domain (the fluid domain except for the fluid below the horizontal plate) consists of two parts, i.e. $\Phi^{*}=\Phi(x, z, t)+\phi(x, z, t)$. The first part corresponds to wave generation, and the second part is associated with vibrations of the plate. With respect to the harmonic generation, both $\Phi$ and $\phi$ are written in a form similar to that in equations (46), namely

$$
\begin{aligned}
& \Phi(x, z, t)=i \omega \Phi(x, z) \exp (i \omega t), \\
& \phi(x, z, t)=i \omega \phi(x, z) \exp (i \omega t) .
\end{aligned}
$$

At the same time, the boundary conditions at the upper fluid surface and the surfaces of the plate read

$$
\begin{aligned}
& -\omega^{2} \Phi(x, z)+g \frac{\partial \Phi(x, z)}{\partial z}+\left.g \frac{\partial \phi(x, z)}{\partial z}\right|_{z=H}=0 \\
& \left.\frac{\partial}{\partial z}[\Phi(x, z)+\phi(x, z)]\right|_{\text {plate }}=\left.\frac{\partial \varphi(x, z)}{\partial z}\right|_{\text {plate }}=W(x) .
\end{aligned}
$$


In order to obtain the first part of the solution, i.e. $\Phi(x, z)$, we resort to a discrete formulation of the problem by means of the finite difference method (FDM). With this method, however, only a finite fluid domain may be considered. And therefore, instead of the infinite fluid layer in Fig. 3, a finite part of it, with a boundary at $x=L$, far off the generator-plate system, is taken into account. With such an approach, however, it is necessary to formulate transmitting boundary conditions at this boundary. Thus, for the steady state, harmonic motion and a sufficiently large distance $L$ from the plate, it is justified to consider only a progressive wave, for which we have

$$
\frac{\partial \Phi}{\partial t}+\frac{\omega}{k_{0}} \frac{\partial \Phi}{\partial x}=0
$$

where

$$
\omega^{2}=g k_{0} \tanh k_{0} h
$$

Equation (62) describes a local boundary condition at the artificial boundary at $x=L$. Since we are dealing with a discrete formulation, it is reasonable to consider a non-local boundary condition, which is more convenient in the discrete method applied. One can show that for the FDM formulation, with vertical spacing of nodal points equal to $a$, the transmitting boundary condition for the velocity potential reads

$$
\left.\Phi\right|_{x=L+a}=-\left.\Phi\right|_{x=L-a}+\left.2 \Phi\right|_{x=L} \cos k_{0} a .
$$

This relation is employed for the difference analogue of Laplace's equation, written for all nodal points at $x=L$. In this way, the problem considered is reduced to unknown nodal values of the potential at points of the finite fluid domain (interior and boundary points of the fluid domain). A solution of the system of FDM equations for the discrete values of the potential $\Phi\left(x_{i}, z_{i}\right)$, where $i$ means the number of a nodal point, depends not only on the generator amplitude, but also on the unknown potential $\phi(x, z)$. These two potentials are coupled through the boundary conditions at the free surface and the surface of the plate (equations 61). In order to find a solution to this problem, a two-step procedure is employed. In the first step, the solution to the potential $\Phi(x, z)$ is expressed in the form of a linear combination of solutions corresponding to unknown parameters $A_{n}(n=1,2, \cdots, n a)$. And then, in the second step, these parameters are obtained by a solution of the plate equation. This, in turn, makes it possible to calculate the deflection of the plate, as well as the pressure field and the amplitude of the free surface elevation.

With respect to the procedure described above, it is necessary to formulate associated boundary conditions for the potential $\Phi^{*}(x, z)$. Thus, at the boundary (1-2) in Fig. 3, one obtains

$$
\begin{aligned}
& \left.\frac{\partial \Phi^{*}}{\partial x}\right|_{1-2}=\frac{\partial \Phi}{\partial x}+\left.\frac{\partial \phi}{\partial x}\right|_{1-2}=\left.\frac{\partial \phi}{\partial x}\right|_{1-2}= \\
& =\sum_{n=1} A_{n} \frac{\cosh r_{n} z}{\sinh r_{n} d}+\sum_{m=1} k_{m}\left[-E_{m}^{1} \tanh k_{m} b+E_{m}^{1} \frac{1}{\tanh k_{m} b}\right] \cos k_{m} z
\end{aligned}
$$


In a similar way,

$$
\left.\frac{\partial \Phi^{*}}{\partial x}\right|_{2-3}=\frac{\partial \Phi}{\partial x}+\left.\frac{\partial \phi}{\partial x}\right|_{2-3}=0,\left.\rightarrow \frac{\partial \Phi}{\partial x}\right|_{2-3}=\left.\frac{\partial \phi}{\partial x}\right|_{2-3}=0 .
$$

At the right boundary at (5-6-7-8), the following relations hold:

$$
\begin{aligned}
& \left.\frac{\partial \Phi^{*}}{\partial x}\right|_{8-7}=\frac{\partial \Phi}{\partial x}+\left.\frac{\partial \phi}{\partial x}\right|_{8-7}=\left.\frac{\partial \phi}{\partial x}\right|_{8-7}= \\
& =\sum_{n=1} A_{n}(-1)^{n} \frac{\cosh r_{n} z}{\sinh r_{n} d}+\sum_{m=1} k_{m}\left[E_{m}^{1} \tanh k_{m} b+E_{m}^{1} \frac{1}{\tanh k_{m} b}\right] \cos k_{m} z
\end{aligned}
$$

and

$$
\left.\frac{\partial \Phi^{*}}{\partial x}\right|_{7-6}=\frac{\partial \Phi}{\partial x}+\left.\frac{\partial \phi}{\partial x}\right|_{7-6}=0,\left.\rightarrow \frac{\partial \Phi}{\partial x}\right|_{7-6}=\left.\frac{\partial \phi}{\partial x}\right|_{7-6}=0 .
$$

At the upper surface of the plate (segment 3-6 in Fig. 3), we have

$$
\left.\frac{\partial \Phi^{*}}{\partial z}\right|_{3-6}=\frac{\partial \Phi}{\partial z}+\left.\frac{\partial \phi}{\partial z}\right|_{3-6}=\left.\frac{\partial \phi}{\partial z}\right|_{3-6}=\sum_{n=1} A_{n} \sin r_{n}(x+b) \cos k_{m} z
$$

The boundary condition at the free surface of the fluid is described by the first equation (61), which is rewritten in the form

$$
\begin{aligned}
& -\omega^{2} \Phi(x, z)+g \frac{\partial \Phi(x, z)}{\partial z}=-\left.g \frac{\partial \phi(x, z)}{\partial z}\right|_{z=H}= \\
& \qquad \begin{array}{l}
\sum_{j=1} C_{j}(-1)^{j+1} \exp \left(-k_{j} x\right), \text { at }(A-4), \quad x \text { - local coordinate } \\
2 \sum_{n=1} A_{n} \frac{e^{-r_{n} h}}{1+e^{-2 r_{n} h}} \sin r_{n}(x+b)+ \\
+\left.\sum_{m=1} k_{m}(-1)^{m+1}\left[D_{m}^{1} \frac{\cosh k_{m} x}{\cosh k_{m} b}+D_{m}^{2} \frac{\sinh k_{m} x}{\sinh k_{m} b}\right]\right|_{(4-5)} \\
\sum_{j=1} B_{j}(-1)^{j+1} \exp \left(-k_{j} x\right), \text { at }(5-E) .
\end{array}
\end{aligned}
$$

The boundary condition at $x=L(D-E)$ describes equation (64). Obviously, at the boundaries $(0-1)$ and $(6-D)$, the normal derivative $(\partial \Phi / \partial z)$ is equal to zero. These boundary conditions depend on the constants $(B, C, D, E)$ that can be expressed in terms of the parameters $A_{n}(n=1,2, \cdots, n a)$. Following the procedure applied, the right-hand side of the discrete system of equations for the potential $\Phi\left(x_{i}, z_{i}\right)$ will depend not only on the generator amplitude, but also on the set of $A_{n}(n=1,2, \cdots, n a)$. These latter parameters will be obtained from equation (1) describing the plate motion. 
With the boundary conditions in mind, the final system of FDM equations for the potential $\Phi\left(x_{i}, z_{i}\right)$ may be written in the following form:

$$
[\boldsymbol{A} \boldsymbol{A}](\Phi)=(\boldsymbol{P}),
$$

where $(\boldsymbol{P})$ depends on the generator amplitude and the potential $\phi(x, z)$. Non-zero components of $(\boldsymbol{P})$, which correspond to nodal points of the free surface, are

$$
P_{r}=-\frac{2 a^{2}}{b^{*}}\left\{\begin{array}{l}
\sum_{j=1} C_{j}(-1)^{j+1} \exp \left(-k_{j} x_{r}\right), \text { at }(A-4), x_{r} \text { - local coordinate } \\
2 \sum_{n=1} A_{n} \frac{e^{-r_{n} h}}{1+e^{-2 r_{n} h}} \sin r_{n}\left(x_{r}+b\right)+ \\
+\left.\sum_{m=1} k_{m}(-1)^{m+1}\left[D_{m}^{1} \frac{\cosh k_{m} x_{r}}{\cosh k_{m} b}+D_{m}^{2} \frac{\sinh k_{m} x_{r}}{\sinh k_{m} b}\right]\right|_{(4-5)} \\
\sum_{j=1} B_{j}(-1)^{j+1} \exp \left(-k_{j} x_{r}\right), \text { at }(5-E) .
\end{array}\right.
$$

The parameters $a$ and $b^{*}$ in this equation denote the horizontal and vertical spacing of nodal points. It is worth adding here that all the constants $\left(B_{j}, C_{j}, \cdots, E_{m}^{1}, E_{m}^{2}\right)$ in this equation are uniquely expressed in terms of $A_{n}(n=1,2, \cdots, n a)$. Finally, in order to find the parameters $A_{n}(n=1,2, \cdots, n a)$, it is necessary to solve the system of equations describing the plate motion:

$$
\begin{aligned}
& A_{n} b\left\{\left[1+\frac{\rho}{m_{p l}} \frac{1}{r_{n}}\left(\frac{1}{\tanh r_{n} d}+\frac{1-e^{-2 r_{n} h}}{1+e^{-2 r_{n} h}}\right)\right]-\frac{D^{*}\left(r_{n}\right)^{4}}{m_{p l} \omega^{2}}\right\}+ \\
& +\frac{\rho}{m_{p l}}\left[E_{0} \int_{-b}^{+b} \sin r_{n}(x+b) d x+\right. \\
& \left.+\sum_{m=1}^{N E}(-1)^{m} \int_{-b}^{+b}\left(E_{m}^{1} \frac{\cosh k_{m} x}{\cosh k_{m} b}+E_{m}^{2} \frac{\sinh k_{m} x}{\sinh k_{m} b}\right) \sin r_{n}(x+b) d x\right]+ \\
& -\frac{\rho}{m_{p l}}\left[\sum_{m=1}^{N D} \int_{-b}^{+b}\left(D_{m}^{1} \frac{\cosh k_{m}^{*} x}{\cosh k_{m}^{*} b}+D_{m}^{2} \frac{\sinh k_{m}^{*} x}{\sinh k_{m}^{*} b}\right) \sin r_{n}(x+b) d x\right]+ \\
& -\frac{\rho}{m_{p l}}\left[\cdots \int_{-b}^{+b} \Phi(x, z=H) \sin r_{n}(x+b) d x \cdot .\right]=0, n=1,2, \cdots, n a
\end{aligned}
$$

The integrals entering these equations are defined by equations (53). Simple, though tedious, manipulations allow us to calculate the set of independent variables $A_{n}(n=1,2, \cdots, n a)$. This solution is illustrated in subsequent Figures 4 and 5.

Plots in Fig. 4 show amplitudes of the free-surface elevation and deflection of the plate. Figure 5 shows the distribution of the maximum deflection of the plate versus 

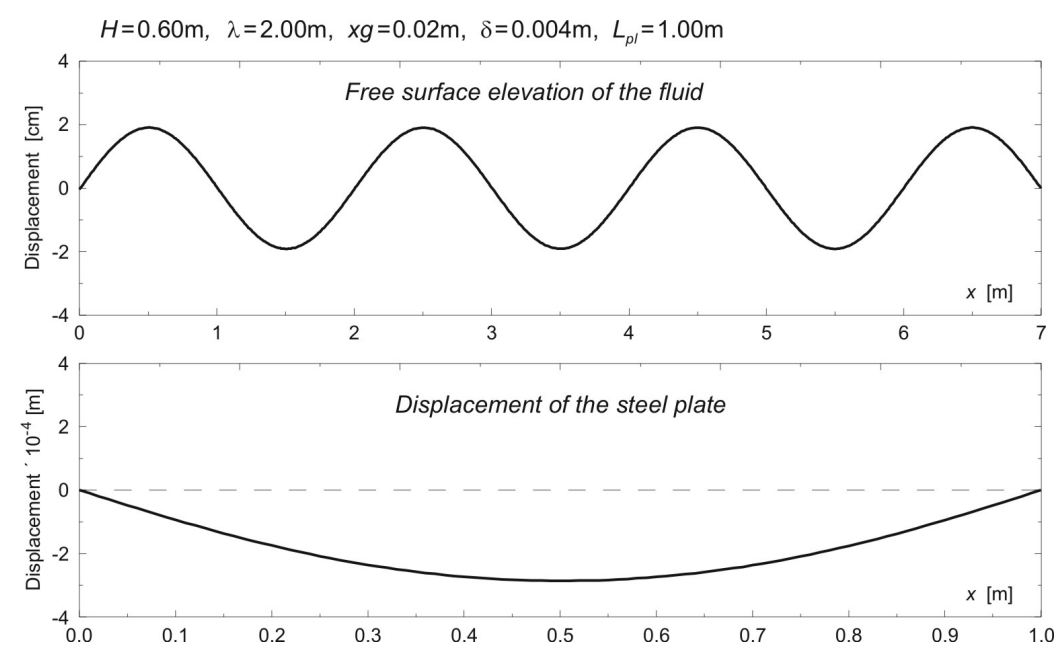

Fig. 4. Free-surface elevation of the fluid and deflection of the steel plate

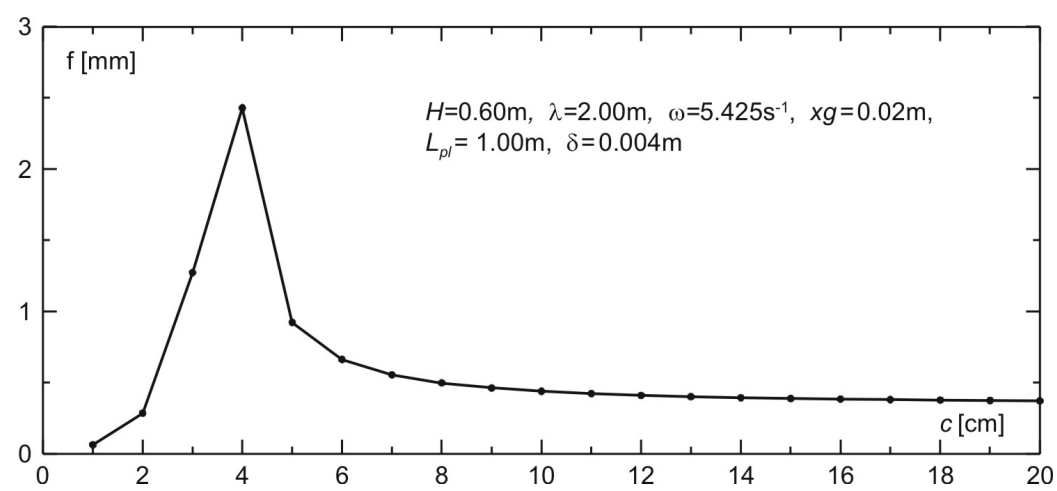

Fig. 5. Maximum deflection of the steel plate versus the gap width

the width of the gap between the plate and the fluid bottom. It should be stressed that this deflection depends on the wave length (associated with the generator frequency), the amplitude of the wave maker, as well as the distance between the plate and the piston generator. Therefore, in practical applications, one should be aware of a certain ambiguity in calculating the plate amplitude, which results from the fundamental assumption of the steady state harmonic motion of the system considered.

\section{Concluding Remarks}

The formulation developed in this paper makes it possible to calculate the co-vibrating mass of fluid and a set of eigenfrequencies of a horizontal thin elastic plate submerged in fluid of constant depth. As compared to vibrations of the plate in air, the most important result of these investigations is an assessment of the reduction in the plate eigenfrequencies due to the co-vibrating mass of fluid. At the same time, the approximate 
theory makes it possible to assess the influence of the gap width on this reduction. It is important to note that the lowest eigenfrequency of the plate vibrations may fall into the range inherent for surface gravitational waves. In such a case, one should be aware of the possibility of a resonance phenomenon that may lead to increased deflection of the plate. Obviously, under natural conditions, one may expect a certain damping of the plate vibrations. Nevertheless, the theoretical result of the possible resonance of waves and plate vibrations is important in the construction of such plates as foundations for offshore structures. At the same time, the numerical experiments conducted for forced vibrations of the plate reveal that, for a certain gap width, one should expect a maximum deflection of the plate. From investigations conducted above, it follows that for a safe operation of such a structure under natural conditions, it may be reasonable to place such a plate foundation at a relatively greater distance from the sea bottom.

\section{References}

Nowacki W. (1972) Dynamics of Structure (in Polish), Arkady, Warszawa.

Kerr A. D. \& Palmer W. T. (1972) The deformation and stresses in floating ice plates, Acta Mechanica, 15.

Sawicki A. (1975) Dynamics of floating roofs (in Polish), Engineering Transactions, 2.

Sawicki A. (1976) Certain problems of hydroelasticity of plates (in Polish), Theoretical and Applied Mechanics, 1 [14], 3-17.

Solecki R. (1966) Vibrations of a floating plate I, Bulletin of the Polish Academy of Sciences, Series Tech. Sc. 11-12, XIV, Warszawa. 